\title{
Positive solutions for elastic beam equations with nonlinear boundary conditions and a parameter
}

\author{
Wenxia Wang*, Yanping Zheng, Hui Yang and Junxia Wang
}

\section{${ }^{*}$ Correspondence:} wwxgg@126.com

Department of Mathematics Taiyuan Normal University, Taiyuan, 030012 , P.R. China

\begin{abstract}
This paper is concerned with the existence, nonexistence, and uniqueness of convex monotone positive solutions of elastic beam equations with a parameter $\lambda$. The boundary conditions mean that the beam is fixed at one end and attached to a bearing device or freed at the other end. By using fixed point theorem of cone expansion, we show that there exists $\lambda^{*} \geq \lambda_{*}>0$ such that the beam equation has at least two, one, and no positive solutions for $0<\lambda \leq \lambda_{*}, \lambda_{*}<\lambda \leq \lambda^{*}$ and $\lambda>\lambda^{*}$, respectively; furthermore, by using cone theory we establish some uniqueness criteria for positive solutions for the beam and show that such solution $x_{\lambda}$ depends continuously on the parameter $\lambda$. In particular, we give an estimate for critical value of parameter $\lambda$.
\end{abstract}

MSC: $34 \mathrm{~B} 18 ; 34 \mathrm{~B} 15$

Keywords: elastic beam equation; positive solution; fixed point; cone

\section{Introduction and preliminaries}

In this paper, we consider the following nonlinear fourth-order two-point boundary value problem (BVP) for elastic beam equation:

$$
\left\{\begin{array}{l}
x^{(4)}(t)=\lambda f(t, x(t)), \quad 0<t<1, \\
x(0)=x^{\prime}(0)=x^{\prime \prime}(1)=x^{\prime \prime \prime}(1)+q(x(1))=0,
\end{array}\right.
$$

where $\lambda \geq 0$ is a parameter. Throughout this paper, we assume that $f \in C\left([0,1] \times R_{+}, R_{+}\right)$, $q \in C\left(R_{+}, R_{+}\right), R_{+}=[0,+\infty) . x \in C[0,1]$ is called a positive solution of BVP (1) if $x$ is a solution of BVP (1) and $x(t)>0,0<t<1$. A convex monotone positive solution means convex nondecreasing positive solution.

Because of characterization of the deformation of the equilibrium state, fourth-order boundary value problems for elastic beam equations are extensively applied to mechanics and engineering; see [1-3]. Some nonlinear elastic beam equations have been studied extensively. For a small sample of such work, we refer the reader to the work of Bai and Wang [4], Bai [5], Bonanno and Bellaa [6], Li [7], Liu and Li [8], Liu [9], Ma and Xu [10], and Ma and Thompson [11] on an elastic beam whose two ends are simply supported, the works of Yang [12] and Zhang [13] on an elastic beam of which one end is embedded and another end is fastened with a sliding clamp, and the work of Graef et al. [14] on multipoint boundary value problems.

\section{焦 Springer}

๑2014 Wang et al.; licensee Springer. This is an Open Access article distributed under the terms of the Creative Commons Attribution License (http://creativecommons.org/licenses/by/2.0), which permits unrestricted use, distribution, and reproduction in any medium, provided the original work is properly cited. 
BVP (1) with $q(x) \equiv 0$ is called a cantilever beam equation, it describes the deflection of the elastic beam fixed at the left end and free at the right end. Existence and multiplicity of positive solutions of cantilever beam problems without parameter have been studied by some authors; see Yao $[15,16]$ and references therein. BVP (1) with $q(x) \not \equiv 0$ describes the deflection of the elastic beam fixed at the left end and attached to a bearing device given by the function $-q$ at the right end. When the elastic beam equation does not contain parameter $\lambda$, the existence of multiple positive solutions and unique positive solution was presented in [17] by variational methods and in [18] by a fixed point theorem, respectively; monotone positive solutions were obtained by using the monotone iteration method in [19]. However, there are few papers concerned with positive solutions for BVP (1) with parameter, especially with the solution's dependence on parameter $\lambda$ in the existing literature. The aim of this paper is to show that the existence and number of convex monotone positive solutions of BVP (1) are affected by the parameter $\lambda$.

The paper is organized as follows. In Section 2, we present that a nontrivial and nonnegative solution of BVP (1) is convex monotone positive solution. In Section 3, we obtain some results on the existence, multiplicity and nonexistence of positive solutions for BVP (1). These results show that the number of positive solutions for BVP (1) depends on the parameter $\lambda$. In Section 4, we establish some uniqueness criteria for positive solutions for BVP (1) and show that such a positive solution $x_{\lambda}$ depends continuously on the parameter $\lambda$. In particular, we give an estimate for the critical value of the parameter $\lambda$.

In the rest of this section, we introduce some notations and known results. For the reader's convenience, we suggest that one refer to [20-22], and [23] for details.

Let $E$ be a real Banach space and $\theta$ denote the zero element of $E$. A nonempty closed convex set $P \subset E$ is called a cone of $E$ if it satisfies (i) $x \in P, r>0 \Rightarrow r x \in P$; (ii) $x \in P$, $-x \in P \Rightarrow x=\theta$. E is partially ordered by the cone $P$, i.e., $x \leq y$ iff $y-x \in P$. A cone $P$ is said to be normal if there exists a positive number $N$, called the normal constant of $P$, such that $\theta \leq x \leq y$ implies $\|x\| \leq N\|y\|$. For $u, v \in E, u \leq v$, denote $[u, v]=\{x \in E \mid u \leq x \leq v\}$.

For all $x, y \in E$, the notation $x \sim y$ means that there exist $\mu_{1}>0$ and $\mu_{2}>0$ such that $\mu_{1} x \leq y \leq \mu_{2} x$. Clearly, $\sim$ is an equivalence relation. Given $e>0$ (i.e., $e \in P$ and $e \neq \theta$ ), we denote by $P_{e}$ the set $P_{e}=\{x \in E \mid x \sim e\}$. It is easy to see that $P_{e} \subset P$.

Let $D \subseteq E$. An operator $T: D \rightarrow E$ is said to be increasing if for $x, y \in D, x \leq y \Rightarrow T x \leq T y$. An element $x^{*} \in D$ is called a fixed point of $T$ if $T x^{*}=x^{*}$.

Lemma 1.1 (Fixed point theorem of cone expansion) [21, 22] Assume that $\Omega_{1}$ and $\Omega_{2}$ are bounded open subsets of $E$ with $\theta \in \Omega_{1} \subset \bar{\Omega}_{1} \subset \Omega_{2}$. Let $T: P \cap\left(\bar{\Omega}_{2}-\Omega_{1}\right) \rightarrow P$ be a completely continuous operator such that $\|T x\| \leq\|x\|$ if $x \in P \cap \partial \Omega_{1}$ and $\|T x\| \geq\|x\|$ if $x \in P \cap \partial \Omega_{2}$. Then $T$ has a fixed point in $P \cap\left(\bar{\Omega}_{2}-\Omega_{1}\right)$.

Lemma 1.2 [23] Let $P$ be a normal cone in $E, T: P_{e} \rightarrow P_{e}$ be increasing and for all $x \in P_{e}$ and $t \in(0,1)$, there exists $\alpha(t) \in(0,1)$ such that $T(t x) \geq t^{\alpha(t)} T x$. Then $T$ has a unique fixed point $x^{*}$ in $P_{e}$. Moreover, constructing successively the sequence $w_{n}=T w_{n-1}(n=1,2, \ldots)$ for any $w_{0} \in P_{e}$, we have $\lim _{n \rightarrow+\infty}\left\|w_{n}-x^{*}\right\|=0$.

\section{Solutions}

In what follows, set $E=C[0,1]$, the Banach space of all continuous functions on $[0,1]$ with the norm $\|x\|=\max \{|x(t)| \mid t \in[0,1]\} . P=\{x \in C[0,1] \mid x(t) \geq 0, t \in[0,1]\}$. It is clear that $P$ is a normal cone and its normality constant is 1 . 
From [18] and [19], it is evident that BVP (1) has an integral formulation given by

$$
x(t)=\lambda \int_{0}^{1} G(t, s) f(s, x(s)) d s+q(x(1))\left(\frac{1}{2} t^{2}-\frac{1}{6} t^{3}\right)
$$

where

$$
G(t, s)=\frac{1}{6} \begin{cases}t^{2}(3 s-t), & 0 \leq t \leq s \leq 1 \\ s^{2}(3 t-s), & 0 \leq s \leq t \leq 1\end{cases}
$$

It is easy to see that $G(t, s) \geq 0$ and

$$
\frac{1}{3} t^{2} s^{2} \leq G(t, s) \leq \frac{1}{2} t^{2} s, \quad t, s \in[0,1]
$$

Define operators $A, B, C_{\lambda}: P \rightarrow C[0,1]$ by

$$
(A x)(t)=\int_{0}^{1} G(t, s) f(s, x(s)) d s, \quad(B x)(t)=q(x(1))\left(\frac{1}{2} t^{2}-\frac{1}{6} t^{3}\right), \quad C_{\lambda}=\lambda A+B .
$$

Then $A(P) \subset P, B(P) \subset P$, and $C_{\lambda}(P) \subset P$.

It is clear from (2) that solving BVP (1) is equivalent to finding fixed points of the operator $C_{\lambda}$. In particular, $x$ is a fixed point of $B$ iff $x$ is a solution of the following BVP:

$$
\left\{\begin{array}{l}
x^{(4)}(t)=0, \quad 0<t<1 \\
x(0)=x^{\prime}(0)=x^{\prime \prime}(1)=x^{\prime \prime \prime}(1)+q(x(1))=0
\end{array}\right.
$$

and $x$ is a fixed point of $\lambda A$ iff $x$ is a solution of the following cantilever beam problem:

$$
\left\{\begin{array}{l}
x^{(4)}(t)=\lambda f(t, x(t)), \quad 0<t<1 \\
x(0)=x^{\prime}(0)=x^{\prime \prime}(1)=x^{\prime \prime \prime}(1)=0 .
\end{array}\right.
$$

Lemma 2.1 If $x \in C^{4}[0,1]$ satisfies

$$
\left\{\begin{array}{l}
x^{(4)}(t) \geq 0, \quad t \in(0,1), \\
x(0)=x^{\prime}(0)=x^{\prime \prime}(1)=0, \quad x^{\prime \prime \prime}(1) \leq 0,
\end{array}\right.
$$

then

(i) $x(t)$ is nondecreasing in $t \in[0,1]$, moreover, $0 \leq x(t) \leq x(1), t \in[0,1]$;

(ii) $x^{\prime \prime}(t) \geq 0, t \in[0,1]$, that is, $x(t)$ is a convex function on $[0,1]$.

Proof From (6), we have $x^{\prime \prime \prime}(t) \leq x^{\prime \prime \prime}(1) \leq 0$. Moreover, $x^{\prime \prime}(t) \geq x^{\prime \prime}(1)=0$. So, $x^{\prime}(t) \geq x^{\prime}(0)=$ 0 . Thus, we complete the proof of the lemma.

Now, let

$$
K=\left\{x \in P \mid x(t) \text { is nondecreasing, } x(t) \geq \frac{2}{3} t^{2} x(1), t \in[0,1]\right\},
$$

then, it is easy to show that $K \subset P$ is also a cone in $E$, and if $x \in K$, then $\|x\|=x(1)$. 
Lemma 2.2 $C_{\lambda}(P) \subset K, A(P) \subset K, B(P) \subset K$.

Proof $x \in P$ implies $x(t) \geq 0$, so $f(t, x(t)) \geq 0$ and $q(x(1)) \geq 0$. Moreover, for $x \in P$,

$$
\begin{aligned}
& \left(C_{\lambda} x\right)^{(4)}(t)=\lambda f(t, x(t)) \geq 0, \quad t \in(0,1), \\
& \left(C_{\lambda} x\right)(0)=\left(C_{\lambda} x\right)^{\prime}(0)=\left(C_{\lambda} x\right)^{\prime \prime}(1)=0, \\
& \left(C_{\lambda} x\right)^{\prime \prime \prime}(1)=-q(x(1)) \leq 0 .
\end{aligned}
$$

By Lemma 2.1, $\left(C_{\lambda} x\right)(t)$ is convex and nondecreasing in $t \in[0,1]$. From (3) and (4) we have

$$
\left(C_{\lambda} x\right)(t)-\frac{2}{3} t^{2}\left(C_{\lambda} x\right)(1) \geq \frac{\lambda}{9} t^{2} \int_{0}^{1} s^{3} f(s, x(s)) d s+q(x(1))\left(\frac{5}{18} t^{2}-\frac{1}{6} t^{3}\right) \geq 0,
$$

that is, $\frac{2}{3} t^{2}\left(C_{\lambda} x\right)(1) \leq\left(C_{\lambda} x\right)(t)$ for $t \in[0,1]$. Thus, we obtain $C_{\lambda}(P) \subset K$. From the above proof, we can show that $A(P) \subset K$ and $B(P) \subset K$. This ends the proof.

\section{Lemma 2.3}

(i) $A: P \rightarrow K$ is a completely continuous operator;

(ii) if $q(x)$ is nondecreasing, then $B: P \rightarrow K$ is a completely continuous operator.

Proof Similarly to the proof of Theorem 1 in [19], applying the Arzela-Ascoli Theorem, the proof can be completed.

From the proof of Lemma 2.2 we can show the following result.

Theorem 2.4 If $x \in P \backslash\{\theta\}$ is a solution for $B V P(1)$, then $x$ is a convex monotone positive solution for $B V P(1)$.

So, in the following sections, we only need to study solutions for BVP (1) in $P \backslash\{\theta\}$.

\section{Existence and nonexistence results}

It is obvious from Lemma 2.2 that if $x \in P \backslash\{\theta\}$ is a solution for BVP (1) then $x \in K \backslash\{\theta\}$. So in this section, we will apply Lemma 1.1 to study the existence, multiplicity and nonexistence of solutions for BVP (1) in $K \backslash\{\theta\}$. It is reasonable that the domain of $C_{\lambda}$ is restricted on $K$. The following conditions will be assumed:

(H1) $f(t, x)$ is nondecreasing in $x \in[0,+\infty)$ for fixed $t \in[0,1]$;

(H2) $q(x)$ is nondecreasing in $x \in[0,+\infty)$;

(H3) $F_{0}:=\int_{0}^{1} s^{2} f(s, 0) d s>0$;

(H4) $q(1)<2$;

(H5) $f_{\infty}:=\lim _{x \rightarrow+\infty} \min _{t \in\left[\frac{1}{2}, 1\right]} \frac{f(t, x)}{x}=+\infty$;

(H6) $q_{\infty}:=\liminf _{x \rightarrow+\infty} \frac{q(x)}{x}>3$.

Set

$$
\Lambda=\left\{\lambda>0 \mid \text { there exists } x_{\lambda} \in K \backslash\{\theta\} \text { such that } C_{\lambda} x_{\lambda}=x_{\lambda}\right\}
$$

and $\lambda^{*}=\sup \Lambda$. 
Lemma 3.1 Suppose that $(\mathrm{H} 1)-(\mathrm{H} 3)$ hold. If $\lambda^{\prime} \in \Lambda$, then $\left(0, \lambda^{\prime}\right] \subset \Lambda$.

Proof $\lambda^{\prime} \in \Lambda$ means that there exists $x_{\lambda^{\prime}} \in K \backslash\{\theta\}$ such that $C_{\lambda^{\prime}} x_{\lambda^{\prime}}=x_{\lambda^{\prime}}$. Therefore, for any $\lambda \in\left(0, \lambda^{\prime}\right]$, we have $C_{\lambda} x_{\lambda^{\prime}} \leq C_{\lambda^{\prime}} x_{\lambda^{\prime}}=x_{\lambda^{\prime}}$. Set $w_{0}=x_{\lambda^{\prime}}, w_{n}=C_{\lambda} w_{n-1}, n=1,2, \ldots$ From $(\mathrm{H} 1)$ and (H2) we obtain $w_{0}(t) \geq w_{1}(t) \geq \cdots \geq w_{n}(t) \geq \cdots \geq \frac{F_{0} \lambda}{3} t^{2}$. By Lemma 2.3 and (H3), $\left\{w_{n}\right\}$ converges to a fixed point of $C_{\lambda}$ in $K \backslash\{\theta\}$. Thus $\left(0, \lambda^{\prime}\right] \subset \Lambda$. This completes the proof.

Let $\lambda_{*}=\frac{2-q(1)}{F_{1}}, F_{1}=\int_{0}^{1} s f(s, 1) d s, u_{0}(t)=\frac{F_{0} \lambda}{3} t^{2}, v_{0}(t)=t^{2}$ and

$$
F_{\infty}=\limsup _{x \rightarrow+\infty} \max _{t \in[0,1]} \frac{f(t, x)}{x}, \quad Q_{\infty}=\limsup _{x \rightarrow+\infty} \frac{q(x)}{x} .
$$

Theorem 3.2 Suppose that (H1)-(H3) hold.

(i) If $(\mathrm{H} 4)$ holds, then $C_{\lambda}$ has minimal and maximal fixed points in $\left[u_{0}, v_{0}\right]$ for $\lambda \in\left(0, \lambda_{*}\right]$. Moreover, there exists $\lambda^{*} \geq \lambda_{*}>0$ such that $C_{\lambda}$ has at least one and has no fixed points in $K \backslash\{\theta\}$ for $0<\lambda<\lambda^{*}$ and $\lambda>\lambda^{*}$, respectively.

(ii) If $F_{\infty}<+\infty, Q_{\infty}<2$, then when $F_{\infty}>0$, there exists $\lambda^{*} \geq \frac{2\left(2-Q_{\infty}\right)}{F_{\infty}}>0$ such that $C_{\lambda}$ has at least one and no fixed points in $K \backslash\{\theta\}$ for $0<\lambda<\lambda^{*}$ and $\lambda>\lambda^{*}$, respectively; when $F_{\infty}=0, C_{\lambda}$ has at least one fixed point in $K \backslash\{\theta\}$ for $\lambda>0$.

Proof (i) From (H1), (H3), and (H4) we have $\lambda_{*}>0$. For any $\lambda \in\left(0, \lambda_{*}\right]$, we obtain

$$
\left(C_{\lambda} u_{0}\right)(t) \geq \frac{\lambda}{3} t^{2} \int_{0}^{1} s^{2} f(s, 0) d s=u_{0}(t), \quad\left(C_{\lambda} v_{0}\right)(t) \leq \frac{1}{2} t^{2}\left(\lambda_{*} F_{1}+q(1)\right) \leq v_{0}(t) .
$$

Set $u_{n}=C_{\lambda} u_{n-1}, v_{n}=C_{\lambda} v_{n-1}, n=1,2, \ldots$, then from (H1) and (H2) we have

$$
u_{0}(t) \leq u_{1}(t) \leq \cdots \leq u_{n}(t) \leq \cdots \leq v_{n}(t) \leq \cdots \leq v_{1}(t) \leq v_{0}(t) .
$$

Lemma 2.3 implies that $\left\{u_{n}\right\}$ and $\left\{v_{n}\right\}$ converge to fixed points $u_{\lambda}$ and $v_{\lambda}$ of $C_{\lambda}$, respectively. From (8) it is evident that $u_{\lambda}, v_{\lambda} \in K \backslash\{\theta\}$ are the minimal fixed point and maximal fixed point of $C_{\lambda}$ in $\left[u_{0}, v_{0}\right]$, respectively. From the definition of $\lambda^{*}$ we can complete the rest of the proof.

(ii) For any $0<\epsilon<2-Q_{\infty}$, there exists $N_{0}>0$ such that $f(t, x) \leq\left(F_{\infty}+\epsilon\right) x$ and $q(x) \leq$ $\left(Q_{\infty}+\epsilon\right) x$ for $x>N_{0}, t \in[0,1]$. Let $w_{0}(t)=2 N_{0} t^{2}$ and $\lambda_{0}=\frac{2\left(2-Q_{\infty}-\epsilon\right)}{F_{\infty}+\epsilon}$, then $\lambda_{0}>0$ and

$$
\left(C_{\lambda_{0}} w_{0}\right)(t) \leq \frac{1}{2} w_{0}(t)\left(\frac{\lambda_{0}}{2}\left(F_{\infty}+\epsilon\right)+Q_{\infty}+\epsilon\right) \leq w_{0}(t)
$$

Similarly to the proof of Lemma 3.1, we can show $\lambda_{0} \in \Lambda$. The conclusion (ii) follows from Lemma 3.1 and the definition of $\lambda^{*}$. This completes the proof of Theorem 3.2.

Lemma 3.3 Suppose that (H1)-(H3) hold and that one of (H5) and (H6) holds. If $\Lambda$ is nonempty, then

(i) $\Lambda$ is bounded from above, that is, $\lambda^{*}<+\infty$;

(ii) $\lambda^{*} \in \Lambda$. 
Proof (i) Suppose to the contrary that there exists an increasing sequence $\left\{\lambda_{n}\right\}_{1}^{+\infty} \subset \Lambda$ such that $\lim _{n \rightarrow+\infty} \lambda_{n}=+\infty$. Set $x_{\lambda_{n}} \in K \backslash\{\theta\}$ is a fixed point of $C_{\lambda_{n}}$, that is, $C_{\lambda_{n}} x_{\lambda_{n}}=x_{\lambda_{n}}$. There are two cases to be considered.

Case 1. $\left\{x_{\lambda_{n}}\right\}_{1}^{+\infty}$ is bounded, that is, there exists a constant $M>0$ such that $\left\|x_{\lambda_{n}}\right\| \leq M$ for $n=1,2, \ldots$. Hence, from (H1), (H3), and (4) we have

$$
M \geq\left\|x_{\lambda_{n}}\right\|=\left(C_{\lambda_{n}} x_{\lambda_{n}}\right)(1) \geq \frac{1}{3} \lambda_{n} \int_{0}^{1} s^{2} f\left(s, x_{\lambda_{n}}(s)\right) d s \geq \frac{F_{0}}{3} \lambda_{n} \rightarrow+\infty,
$$

which is a contradiction.

Case 2. $\left\{x_{\lambda_{n}}\right\}_{1}^{+\infty}$ is unbounded, that is, there exists a subsequence of $\left\{x_{\lambda_{n}}\right\}_{1}^{+\infty}$, still denoted by $\left\{x_{\lambda_{n}}\right\}_{1}^{+\infty}$, such that $\lim _{n \rightarrow+\infty}\left\|x_{\lambda_{n}}\right\|=+\infty$.

When (H5) holds, take $L>\frac{72}{\lambda_{1}}$, there exists $N_{1}>0$ such that $f(t, x) \geq L x$ for $x \geq N_{1}$, $t \in\left[\frac{1}{2}, 1\right]$. Choose $n_{1}$ such that $\left\|x_{\lambda_{n_{1}}}\right\|>6 N_{1}$. Thus, $f\left(t, \frac{1}{6}\left\|x_{\lambda_{n_{1}}}\right\|\right) \geq \frac{1}{6} L\left\|x_{\lambda_{n_{1}}}\right\|, t \in\left[\frac{1}{2}, 1\right]$. Moreover, from (H1) and the definition of $K$, we have

$$
\left\|x_{\lambda_{n_{1}}}\right\|=\left(C_{\lambda_{n_{1}}} x_{\lambda_{n_{1}}}\right)(1) \geq \frac{1}{3} \lambda_{1} \int_{\frac{1}{2}}^{1} s^{2} f\left(s, \frac{1}{6}\left\|x_{\lambda_{n_{1}}}\right\|\right) d s>\frac{1}{72} \lambda_{1} L\left\|x_{\lambda_{n_{1}}}\right\|>\left\|x_{\lambda_{n_{1}}}\right\|,
$$

which is a contradiction.

When (H6) holds, choose $\epsilon>0$ such that $\frac{1}{3}\left(q_{\infty}-\epsilon\right)>1$. There exists $N_{2}>1$ such that $q(x) \geq\left(q_{\infty}-\epsilon\right) x$ for $x \geq N_{2}$. Choose $n_{2}$ such that $\left\|x_{\lambda_{n_{2}}}\right\|>N_{2}$, so

$$
q\left(x_{\lambda_{n_{2}}}(1)\right)=q\left(\left\|x_{\lambda_{n_{2}}}\right\|\right) \geq\left(q_{\infty}-\epsilon\right)\left\|x_{\lambda_{n_{2}}}\right\| .
$$

Moreover,

$$
\left\|x_{\lambda_{n_{2}}}\right\|=\left(C_{\lambda_{n_{2}}} x_{\lambda_{n_{2}}}\right)(1) \geq \frac{1}{3} q\left(x_{\lambda_{n_{2}}}(1)\right) \geq \frac{1}{3}\left(q_{\infty}-\epsilon\right)\left\|x_{\lambda_{n_{2}}}\right\|>\left\|x_{\lambda_{n_{2}}}\right\|,
$$

which is a contradiction.

Consequently, we find that $\Lambda$ is bounded from above.

(ii) By the definition of $\lambda^{*}$, there exists a nondecreasing sequence $\left\{\lambda_{n}\right\}_{1}^{+\infty}$ such that $\lim _{n \rightarrow+\infty} \lambda_{n}=\lambda^{*}$. Let $x_{\lambda_{n}} \in K \backslash\{\theta\}$ be a fixed point of $C_{\lambda_{n}}$. Arguing similarly as above in case 2, we can show that $\left\{x_{\lambda_{n}}\right\}_{1}^{+\infty}$ is a bounded subset in $K$, that is, there exists a constant $M>0$ such that $\left\|x_{\lambda_{n}}\right\| \leq M, n=1,2, \ldots$; on the other hand, note that

$$
\left|x_{\lambda_{n}}\left(t_{1}\right)-x_{\lambda_{n}}\left(t_{2}\right)\right| \leq \lambda^{*} \int_{0}^{1}\left|G\left(t_{1}, s\right)-G\left(t_{2}, s\right)\right| f(s, M) d s+\frac{4}{3} q(M)\left|t_{1}-t_{2}\right|
$$

we see that $\left\{x_{\lambda_{n}}\right\}_{1}^{+\infty}$ is an equicontinuous subset in $K$. Consequently, by an application of the Arzela-Ascoli Theorem we conclude that $\left\{x_{\lambda_{n}}\right\}_{1}^{+\infty}$ is a relatively compact set in $K$. So, there exists a subsequence $\left\{x_{\lambda_{i}}\right\} \subset\left\{x_{\lambda_{n}}\right\}$ converging to $x^{*} \in K$. Note that

$$
x_{\lambda_{n_{i}}}(t)=\lambda_{n_{i}} \int_{0}^{1} G(t, s) f\left(s, x_{\lambda_{n_{i}}}(s)\right) d s+q\left(x_{\lambda_{n_{i}}}(1)\right)\left(\frac{1}{2} t^{2}-\frac{1}{6} t^{3}\right) .
$$

By taking the limit we have $x^{*}(t)=\left(C_{\lambda^{*}} x^{*}\right)(t) \geq \frac{\lambda_{1}}{3} F_{0} t^{2}$, that is, $\lambda^{*} \in \Lambda$. The proof is complete. 
Theorem 3.4 Suppose that (H1)-(H4) hold and that one of (H5) and (H6) holds. Then, there exists $a \lambda^{*} \geq \lambda_{*}>0$ such that $B V P$ (1) has at least two, one, and no positive solutions for $0<\lambda \leq \lambda_{*}, \lambda_{*}<\lambda \leq \lambda^{*}$ and $\lambda>\lambda^{*}$, respectively.

Proof Theorem 3.2 implies $\left(0, \lambda_{*}\right] \subset \Lambda$, so $\lambda^{*} \geq \lambda_{*}>0$. From Lemmas 3.1 and 3.3, we have $\left(0, \lambda^{*}\right]=\Lambda$. Therefore, from the definition of $\lambda^{*}$ we only to prove that $C_{\lambda}$ has at least two fixed points in $K \backslash\{\theta\}$ for $\lambda \in\left(0, \lambda_{*}\right]$.

Now, given $\lambda \in\left(0, \lambda_{*}\right]$. Theorem 3.2 means that $C_{\lambda}$ has at least one fixed point $x_{\lambda, 1} \in$ $K \backslash\{\theta\}$ which satisfies $\left\|x_{\lambda, 1}\right\| \leq 1$.

Let $K_{1}=\{x \in K \mid\|x\|<1\}$. Note that $t(3-t) \leq 2$ for $t \in[0,1]$, so for $x \in K$ with $\|x\|=1$, i.e., $x \in \partial K_{1}$, we have

$$
\left\|C_{\lambda} x\right\|=\left(C_{\lambda} x\right)(1) \leq \frac{1}{6}\left(\lambda_{*} \int_{0}^{1} s^{2}(3-s) f(s, 1) d s+2 q(1)\right) \leq \frac{2}{3}<\|x\| .
$$

When (H5) holds, take $L^{\prime}>\frac{72}{\lambda}$, there exists $N_{1}^{\prime}>1$ such that $f(t, x) \geq L^{\prime} x$ for $x \geq N_{1}^{\prime}$, $t \in\left[\frac{1}{2}, 1\right]$. Set $K_{2}=\left\{x \in K \mid\|x\|<6 N_{1}^{\prime}\right\}$. Then $\bar{K}_{1} \subset K_{2}$. If $x \in \partial K_{2}$, we have

$$
\left\|C_{\lambda} x\right\|=\left(C_{\lambda} x\right)(1) \geq \frac{\lambda}{3} \int_{\frac{1}{2}}^{1} s^{2} f\left(s, \frac{1}{6}\|x\|\right) d s>\frac{\lambda L^{\prime}}{72}\|x\|>\|x\| .
$$

When (H6) holds, from the proof of Lemma 3.3 we can set $K_{2}^{\prime}=\left\{x \in K \mid\|x\|<N_{2}\right\}$. Then $\bar{K}_{1} \subset K_{2}^{\prime}$. If $x \in \partial K_{2}^{\prime}$, we have $\left\|C_{\lambda} x\right\|=C_{\lambda} x(1) \geq \frac{1}{3} q(x(1)) \geq \frac{1}{3}\left(q_{\infty}-\epsilon\right) x(1)>\|x\|$.

Consequently, in virtue of Lemma 1.1 we find that $C_{\lambda}$ has another fixed point $x_{\lambda, 2}$ with

$$
x_{\lambda, 2} \in \begin{cases}\bar{K}_{2}-K_{1}, & \text { as }(\mathrm{H} 5) \text { holds } \\ \bar{K}_{2}^{\prime}-K_{1}, & \text { as (H6) holds }\end{cases}
$$

Equation (9) implies that $C_{\lambda}$ has no fixed points in $\partial K_{1}$. In conclusion, for $\lambda \in\left(0, \lambda_{*}\right], C_{\lambda}$ has at least two fixed points $x_{\lambda, 1}$ and $x_{\lambda, 2}$ in $K$ with $0<\left\|x_{\lambda, 1}\right\|<1<\left\|x_{\lambda, 2}\right\|$. The proof is complete.

Remark 3.1 In the above results, we can replace (H5) with the following condition: there exists $\epsilon_{0} \in(0,1)$ such that $\lim _{x \rightarrow+\infty} \min _{t \in\left[\epsilon_{0}, 1\right]} \frac{f(t, x)}{x}=+\infty$.

In the following, we give some sufficient conditions that BVP (1) has no positive solutions.

Theorem 3.5 Suppose that there exists a nonnegative integrable function a $(t)$ such that $f(t, x) \geq a(t) x, t \in[0,1], x \in[0,+\infty)$ and $a^{*}:=\int_{0}^{1} s^{4}(3-s) a(s) d s>0$. Then $B V P(1)$ has no positive solutions for $\lambda>\frac{9}{a^{*}}$.

Proof Assume to the contrary that $x_{\lambda} \in K \backslash\{\theta\}$ is a solution of BVP (1), then $\left\|x_{\lambda}\right\|=$ $\left(C_{\lambda} x_{\lambda}\right)(1) \geq \frac{1}{9}\left\|x_{\lambda}\right\| \lambda \int_{0}^{1} s^{4}(3-s) a(s) d s>\left\|x_{\lambda}\right\|$, which is a contradiction. The proof is complete.

Similarly to the proof of Theorem 3.5, we can easily obtain the following results. 
Theorem 3.6 Suppose that there exist an integrable function $a_{1}(t) \geq 0$ and a number $b \in[0,3)$ such that $f(t, x) \leq a_{1}(t) x, q(x) \leq b x, t \in[0,1], x \in[0,+\infty)$ and $a_{1}^{*}:=\int_{0}^{1} s^{2}(3-$ s) $a_{1}(s) d s>0$. Then BVP (1) has no positive solutions for $0 \leq \lambda<\frac{6-2 b}{a_{1}^{*}}$.

Theorem 3.7 Suppose that $q(x)>3 x, x \in[0,+\infty)$. Then $B V P(1)$ has no positive solutions for $\lambda \geq 0$.

Remark 3.2 When $q(x) \equiv 0$, BVP (1) becomes a cantilever beam problem (5). In this case, we can delete the conditions on $q$ in Theorems 3.2, 3.4-3.6 and obtain the following corresponding results for BVP (5).

Suppose that (H1) and (H3) hold. Then BVP (5) has minimal and maximal solutions in $\left[u_{0}, v_{0}\right]$ for $\lambda \in\left(0, \frac{2}{F_{1}}\right]$. Further, if $0<F_{\infty}<+\infty$, then there exists $\lambda^{*} \geq \max \left\{\frac{2}{F_{1}}, \frac{4}{F_{\infty}}\right\}$ such that BVP (5) has at least one and has no positive solutions for $0<\lambda<\lambda^{*}$ and $\lambda>\lambda^{*}$, respectively; if $F_{\infty}=0$ then BVP (5) has at least one positive solution for $\lambda>0$.

Suppose that (H1), (H3), and (H5) hold. Then $\lambda^{*} \geq \frac{2}{F_{1}}$ and BVP (5) has at least two, one and has no positive solutions for $0<\lambda \leq \frac{2}{F_{1}}, \frac{2}{F_{1}}<\lambda \leq \lambda^{*}$ and $\lambda>\lambda^{*}$, respectively.

Under the conditions in Theorem 3.5, BVP (5) has no positive solutions for $\lambda>\frac{9}{a^{*}}$.

Suppose that $a_{1}(t)$ and $a_{1}^{*}$ satisfy the conditions in Theorem 3.6, then BVP (5) has no positive solutions for $0 \leq \lambda<\frac{6}{a_{1}^{*}}$.

Remark 3.3 (i) We give an example to illustrate Theorem 3.2. Let $f(t, x)=t^{3}+\frac{1}{5} \ln (1+x)$, and

$$
q(x)= \begin{cases}\sin x, & 0 \leq x \leq \frac{\pi}{2} \\ \frac{2}{\pi} x, & \frac{\pi}{2} \leq x \leq \pi, \frac{3 \pi}{2} \leq x \leq 2 \pi \\ 2+|\sin x|, & \pi \leq x \leq \frac{3 \pi}{2} \\ 4, & x \geq 2 \pi\end{cases}
$$

By straightforward calculations we see that $F_{0}=\frac{1}{6}, F_{1}=\frac{1}{5}\left(1+\frac{\ln 2}{2}\right), q(1)=\sin 1, \lambda_{*}=\frac{2-q(1)}{F_{1}} \doteq$ 7.361, $F_{\infty}=\frac{1}{5}$, and $Q_{\infty}=0$. So the conditions in Theorem 3.2 are satisfied. Therefore, by Theorem 3.2 we find that there exists $\lambda^{*} \geq \frac{4}{F_{\infty}}=20$ such that BVP (1) has minimal and maximal solutions in $\left[u_{0}, v_{0}\right]$ for $0<\lambda \leq 7.361$, has at least one positive solution for $7.361<\lambda<\lambda^{*}$ and has no positive solutions for $\lambda>\lambda^{*}$, where $u_{0}(t)=\frac{\lambda}{18} t^{2}$ and $v_{0}(t)=t^{2}$.

We give another example to illustrate Theorem 3.4. Let $f(t, x)=\frac{t}{2}\left(1+x^{2}\right)+\frac{1}{8} e^{t} \sqrt{x}$, and

$$
q(x)= \begin{cases}\frac{1}{8} x^{\frac{4}{3}}, & 0 \leq x \leq 8 \\ 2, & x \geq 8\end{cases}
$$

A straightforward calculation can show that $F_{0}=\frac{1}{8}, f_{\infty}=+\infty, q(1)=\frac{1}{8}, F_{1}=\frac{11}{24}$, and $\lambda_{*}=\frac{45}{11}$ Therefore, the conditions (H1)-(H5) hold. Thus, by Theorem 3.4 we see that there exists $\lambda^{*} \geq \frac{45}{11}$ such that BVP (1) has at least two, one, and no positive solutions for $0<\lambda \leq \frac{45}{11}$, $\frac{45}{11}<\lambda \leq \lambda^{*}$, and $\lambda>\lambda^{*}$, respectively.

(ii) In Theorems 3.5-3.7, we do not require $f$ and $q$ to be monotone in $x$. For example, let $f(t, x)=\frac{t x}{1+|\cos x|}$ and

$$
q(x)= \begin{cases}x|\sin x|, & 0 \leq x \leq 2 \pi \\ 0, & x \geq 2 \pi\end{cases}
$$


Take $a_{1}(t)=t, b=1$, then the conditions in Theorem 3.6 are satisfied and $a_{1}^{*}=\frac{11}{20}$. So by Theorem 3.6 we find that BVP (1) has no positive solutions for $0 \leq \lambda<\frac{80}{11}$.

\section{Uniqueness and dependence on parameter}

In this section, we will apply cone theory to further study the uniqueness of solution for BVP (1) in $P \backslash\{\theta\}$ and the dependence of such a positive solution on the parameter $\lambda$. The following hypotheses are needed:

(H7) $q(1) \neq 0$ and for all $x \in[0,+\infty)$ and $r \in(0,1)$, there exists $\alpha(r) \in(0,1)$ such that $q(r x) \geq r^{\alpha(r)} q(x)$

(H8) $f(t, 1) \not \equiv 0$ and $f(t, r x) \geq r f(t, x)$ for $r \in(0,1), t \in[0,1], x \in[0,+\infty)$;

(H9) for all $t \in[0,1], x \in[0,+\infty)$ and $r \in(0,1)$, there exists $\beta(r) \in(0,1)$ such that $f(t, r x) \geq r^{\beta(r)} f(t, x)$.

Remark 4.1 The inequalities in (H7), (H8), and (H9) are equivalent to the following inequalities, respectively:

$$
\begin{aligned}
& q(s x) \leq s^{\alpha\left(\frac{1}{s}\right)} q(x), \quad s>1, x \in[0,+\infty), \\
& f(t, s x) \leq s f(t, x), \quad s>1, t \in[0,1], x \in[0,+\infty), \\
& f(t, s x) \leq s^{\beta\left(\frac{1}{s}\right)} f(t, x), \quad s>1, t \in[0,1], x \in[0,+\infty) .
\end{aligned}
$$

Let $e(t)=t^{2}$ and define $P_{e}$ as in Section 1. It is obvious that $P_{e} \subset P$ and if $x \in P_{e}$ then $x(0)=0$ and $x(t)>0, t \in(0,1]$.

Remark 4.2 (H2) and (H7) imply $q(x)>0$ for $x>0$. Moreover, $q(x(1))>0$ for $x \in P_{e}$.

Remark 4.3 Let $x_{\lambda}$ be a solution for BVP (1) in $P \backslash\{\theta\}$. If (H2) and (H7) hold, then $x_{\lambda} \in P_{e}$. Indeed, from Theorem 2.4 we have $x_{\lambda}(1)=\left\|x_{\lambda}\right\|$. So Remark 4.2 implies $q\left(x_{\lambda}(1)\right)>0$. Note that

$$
\frac{q\left(x_{\lambda}(1)\right)}{3} t^{2} \leq x_{\lambda}(t)=\left(C_{\lambda} x_{\lambda}\right)(t) \leq \frac{1}{2}\left(\lambda \int_{0}^{1} s f\left(s, x_{\lambda}(s)\right) d s+q\left(x_{\lambda}(1)\right)\right) t^{2},
$$

we conclude $x_{\lambda} \in P_{e}$.

So, in this section, we only need to consider the unique solution for BVP (1) in $P_{e}$.

Lemma 4.1 Assume that $(\mathrm{H} 2)$ and $(\mathrm{H} 7)$ hold. Then $B$ has a unique fixed point $x_{0}$ in $P_{e}$, moreover, constructing successively the sequence $w_{n}=B w_{n-1}(n=1,2, \ldots)$ for any initial value $w_{0} \in P_{e}$, we have $\lim _{n \rightarrow+\infty}\left\|w_{n}-x_{0}\right\|=0$.

Proof For any $x \in P_{e}$, we have $\frac{1}{3} q(x(1)) t^{2} \leq B x(t) \leq \frac{1}{2} q(x(1)) t^{2}$, which means $B\left(P_{e}\right) \subset P_{e}$. For all $x \in P_{e}, r \in(0,1)$, from (H7) we have $B(r x)(t) \geq r^{\alpha(r)} B x(t)$. Consequently, the conclusion follows from Lemma 1.2. This completes the proof.

Lemma 4.2 Assume that (H1), (H2), (H7), and (H8) hold. Then

(i) $C_{\lambda}: P_{e} \rightarrow P_{e}$ is an increasing operator; 
(ii) for any $\lambda \geq 0$ and $x \in P_{e}$, there exists $\varphi(\lambda, x) \in(0,1)$ such that $B x \geq \varphi(\lambda, x) C_{\lambda} x$;

(iii) for $[u, v] \subset P_{e}$ and $r \in(0,1)$, there exists $\eta(r, u, v)>0$ such that

$$
C_{\lambda}(r x) \geq r(1+\eta(r, u, v)) C_{\lambda} x, \quad \forall x \in[u, v]
$$

Proof The conclusion (i) follows from (H1), (H2), (H7), and (4).

The proof of (ii). For given $\lambda \geq 0, x \in P_{e}$, from (H1) and (4) we have

$$
\left(C_{\lambda} x\right)(t) \leq \frac{1}{2} t^{2}\left(\lambda \int_{0}^{1} s f(s,\|x\|) d s+q(x(1))\right) \leq \frac{3\left(\lambda \int_{0}^{1} s f(s,\|x\|) d s+q(x(1))\right.}{2 q(x(1))} B x(t) .
$$

Let

$$
\varphi(\lambda, x)=\frac{2 q(x(1))}{3\left(\lambda \int_{0}^{1} s f(s,\|x\|) d s+q(x(1))\right)},
$$

then $0<\varphi(\lambda, x)<1$ and

$$
B x \geq \varphi(\lambda, x) C_{\lambda} x .
$$

The proof of (iii). For any $x \in[u, v], u, v \in P_{e}$, from (10) and (11) we have

$$
B x \geq \frac{2 q(x(1))}{3\left(\lambda \int_{0}^{1} s f(s,\|x\|) d s+q(x(1))\right.} C_{\lambda} x \geq \frac{2 q(u(1))}{3\left(\lambda \int_{0}^{1} s f(s,\|v\|) d s+q(v(1))\right)} C_{\lambda} x .
$$

Moreover, from (H7) and (H8) we have

$$
C_{\lambda}(r x) \geq \lambda r A x+r^{\alpha(r)} B x \geq r(1+\eta(r, u, v)) C_{\lambda} x, \quad \forall r \in(0,1), x \in[u, v],
$$

where $\eta(r, u, v)=\frac{2\left(r^{\alpha(r)}-r\right) q(u(1))}{3 r\left(\lambda \int_{0}^{1} s f(s,\|v\|) d s+q(v(1))\right)}>0$. This completes the proof.

Lemma 4.3 Assume that (H1), (H2), (H7), and (H8) hold. Then $C_{\lambda}$ has a unique fixed point $x_{\lambda}$ in $P_{e}$ iff there exists $y_{\lambda} \in P_{e}$ such that $C_{\lambda} y_{\lambda} \leq y_{\lambda}$. Moreover, constructing successively the sequence $w_{n}=C_{\lambda} w_{n-1}(n=1,2, \ldots)$ for any initial value $w_{0} \in P_{e}$, we have

$$
\lim _{n \rightarrow+\infty}\left\|w_{n}-x_{\lambda}\right\|=0
$$

Proof ' $\Rightarrow$ ' Let $x_{\lambda}$ be a fixed point of $C_{\lambda}$ in $P_{e}$, i.e., $C_{\lambda} x_{\lambda}=x_{\lambda}$. Taking $y_{\lambda}=x_{\lambda}$, we obtain $C_{\lambda} y_{\lambda} \leq y_{\lambda}$.

' $\Leftarrow$ 'By virtue of Lemma 4.1, $B$ has a unique fixed point $x_{0}$ in $P_{e}$. Moreover,

$$
x_{0} \leq C_{\lambda} x_{0}, \quad \lambda \geq 0 .
$$

Now, we are going to prove

$$
x_{0} \leq y_{\lambda}, \quad \lambda \geq 0 .
$$


Let $\tau_{0}=\inf \left\{\tau>0 \mid x_{0} \leq \tau y_{\lambda}\right\}$, then $\tau_{0} \leq 1$. Otherwise, $\tau_{0}>1$, from Lemma 4.2 we have

$$
x_{0} \leq C_{\lambda} x_{0} \leq C_{\lambda}\left(\tau_{0} y_{\lambda}\right) \leq \frac{\tau_{0}}{1+\eta\left(\frac{1}{\tau_{0}}, x_{0}, \tau_{0} y_{\lambda}\right)} C_{\lambda} y_{\lambda} \leq \frac{\tau_{0}}{1+\eta\left(\frac{1}{\tau_{0}}, x_{0}, \tau_{0} y_{\lambda}\right)} y_{\lambda} .
$$

By the definition of $\tau_{0}$, we get a contradiction $\tau_{0} \leq \frac{\tau_{0}}{1+\eta\left(\frac{1}{\tau_{0}}, x_{0}, \tau_{0} y_{\lambda}\right)}$. Thus, (14) holds.

Set $x_{n}=C_{\lambda} x_{n-1}, y_{n}=C_{\lambda} y_{n-1}, y_{0}=y_{\lambda}, n=1,2, \ldots$ From (13) and (14) we have

$$
x_{0} \leq x_{1} \leq \cdots \leq x_{n} \leq \cdots \leq y_{n} \leq \cdots \leq y_{1} \leq y_{0}=y_{\lambda} .
$$

Lemma 2.3 implies that $\left\{x_{n}\right\}$ and $\left\{y_{n}\right\}$ converge to fixed points $x^{*}$ and $y^{*}$ of $C_{\lambda}$, respectively. From (15), we have

$$
x_{0} \leq x_{1} \leq \cdots \leq x_{n} \leq \cdots \leq x^{*} \leq y^{*} \leq \cdots \leq y_{n} \leq \cdots \leq y_{1} \leq y_{\lambda}
$$

To prove that $C_{\lambda}$ has only one fixed point in $\left[x_{0}, y_{\lambda}\right]$, let

$$
\mu_{n}=\sup \left\{\tau>0 \mid x_{n} \geq \tau y_{n}\right\}, \quad n=0,1,2, \ldots,
$$

then

$$
0<\mu_{n} \leq 1, \quad x_{n} \geq \mu_{n} y_{n}, \quad n=1,2, \ldots
$$

From (16)-(18) we infer that $0<\mu_{0} \leq \mu_{1} \leq \cdots \leq \mu_{n} \leq \cdots \leq 1$, which means that $\lim _{n \rightarrow+\infty} \mu_{n}=\mu \leq 1$. We assert that $\mu=1$. Otherwise, $0<\mu_{n} \leq \mu<1$ for $n \geq 1$, then by Lemma 4.2 we deduce that

$$
x_{n+1} \geq C_{\lambda}\left(\mu_{n} y_{n}\right) \geq C_{\lambda}\left(\frac{\mu_{n}}{\mu} \mu y_{n}\right) \geq \frac{\mu_{n}}{\mu} C_{\lambda}\left(\mu y_{n}\right) \geq \mu_{n}\left(1+\eta\left(\mu, x_{0}, y_{\lambda}\right)\right) y_{n+1} \text {. }
$$

By (17), we have $\mu_{n+1} \geq \mu_{n}\left(1+\eta\left(\mu, x_{0}, y_{\lambda}\right)\right)$, moreover, $\mu \geq \mu\left(1+\eta\left(\mu, x_{0}, y_{\lambda}\right)\right)$, which is a contradiction. So $\mu=1$. Thus, by (16) and (18) we have

$$
\left\|y^{*}-x^{*}\right\| \leq\left\|y_{n}-x_{n}\right\| \leq\left(1-\mu_{n}\right)\left\|y_{0}\right\| \rightarrow 0 \quad \text { as } n \rightarrow+\infty
$$

which means that $x^{*}=y^{*}:=x_{\lambda}$ is the unique fixed point of $C_{\lambda}$ in $\left[x_{0}, y_{\lambda}\right]$.

Now, we prove that $x^{*}$ is the unique fixed point of $C_{\lambda}$ in $P_{e}$. By the above proof, we only need to show that $C_{\lambda}$ does not have any fixed point in $P_{e} \backslash\left[x_{0}, y_{\lambda}\right]$. If $\bar{x}$ is a fixed point of $C_{\lambda}$ in $P_{e} \backslash\left[x_{0}, y_{\lambda}\right]$. Let

$$
\bar{\mu}=\sup \left\{\tau>0 \mid \tau x^{*} \leq \bar{x} \leq \frac{1}{\tau} x^{*}\right\} .
$$

It is evident that $0<\bar{\mu} \leq 1$. If $0<\bar{\mu}<1$, then $x_{0} \leq x^{*} \leq \frac{1}{\bar{\mu}} x^{*} \leq \frac{1}{\bar{\mu}} y_{\lambda}$. By Lemma 4.2 we have

$$
\begin{aligned}
\bar{\mu}\left(1+\eta\left(\bar{\mu}, x_{0}, \frac{1}{\bar{\mu}} y_{\lambda}\right)\right) x^{*} & \leq C_{\lambda}\left(\bar{\mu} x^{*}\right) \leq \bar{x}=C_{\lambda} \bar{x} \leq C_{\lambda}\left(\frac{1}{\bar{\mu}} x^{*}\right) \\
& \leq \frac{1}{\bar{\mu}\left(1+\eta\left(\bar{\mu}, x_{0}, \frac{1}{\bar{\mu}} y_{\lambda}\right)\right)} x^{*} .
\end{aligned}
$$


Thus, from (19) we have $\bar{\mu} \geq \bar{\mu}\left(1+\eta\left(\bar{\mu}, x_{0}, \frac{1}{\bar{\mu}} y_{\lambda}\right)\right)$, which is a contradiction. So $\bar{\mu}=1$. Moreover, $\bar{x}=x^{*}$, which implies the contradiction: $\bar{x}=x^{*} \in\left[x_{0}, y_{\lambda}\right]$ and $\bar{x} \in P_{e} \backslash\left[x_{0}, y_{\lambda}\right]$.

Finally, the iterative scheme and (12) can be proved in a similar way to the proof of Theorem 3.4 of [21], here it is omitted. The proof is complete.

Theorem 4.4 Assume that (H1), (H2), (H7), and (H8) hold. Then there exists a $\lambda^{*}>0$ such that $B V P(1)$ has a unique solution $x_{\lambda}$ in $P_{e}$ for $\lambda \in\left[0, \lambda^{*}\right)$ and does not have any solution in $P_{e}$ for $\lambda \geq \lambda^{*}$. Moreover, set $w_{n}=\lambda A w_{n-1}+B w_{n-1}(n=1,2, \ldots)$ for any $w_{0} \in P_{e}$, then (12) holds.

Proof By Lemma 4.1, $B$ has the unique fixed point $x_{0}$ in $P_{e}$. So $x_{0}(t)=q\left(x_{0}(1)\right)\left(\frac{1}{2} t^{2}-\frac{1}{6} t^{3}\right)$, moreover, $\left\|x_{0}\right\|=x_{0}(1)=\frac{1}{3} q\left(x_{0}(1)\right)>0$. Let $\rho_{0}=\frac{1}{2} \int_{0}^{1} \frac{s f\left(s, x_{0}(1)\right)}{x_{0}(1)} d s$, we have

$$
\left(A x_{0}\right)(t) \leq \frac{t^{2}}{2} \int_{0}^{1} s f\left(s, x_{0}(1)\right) d s \leq q\left(x_{0}(1)\right)\left(\frac{1}{2} t^{2}-\frac{1}{6} t^{3}\right) \rho_{0}=\rho_{0} x_{0}(t) .
$$

Set $\Delta=\left\{\lambda \geq 0 \mid\right.$ there exists $x_{\lambda} \in P_{e}$ such that $\left.C_{\lambda} x_{\lambda}=x_{\lambda}\right\}$. Lemma 4.3 implies that

$$
\Delta=\left\{\lambda \geq 0 \mid \text { there exists } y_{\lambda} \in P_{e} \text { such that } C_{\lambda} y_{\lambda} \leq y_{\lambda}\right\}
$$

Similarly to the proof of Lemma 3.1, we can show that $\lambda \in \Delta$ implies $[0, \lambda] \subset \Delta$.

Now, take $s_{0}>1$ and let $\lambda_{0}=\frac{1}{\rho_{0}}\left(1-s_{0}^{\alpha\left(\frac{1}{s_{0}}\right)-1}\right)$ and $y_{\lambda_{0}}=s_{0} x_{0}$, then $\lambda_{0}>0$ and $y_{\lambda_{0}} \in P_{e}$. By (H7), (H8), and (20), we have $C_{\lambda_{0}} y_{\lambda_{0}} \leq \lambda_{0} s_{0} \rho_{0} x_{0}+s_{0}^{\alpha\left(\frac{1}{s_{0}}\right)} x_{0} \leq y_{\lambda_{0}}$, that is, $\lambda_{0} \in \Delta$. Moreover, $\left[0, \lambda_{0}\right] \subset \Delta$.

Let $\lambda^{*}=\sup \Delta$, then $\lambda^{*} \geq \lambda_{0}>0$. We assert that $\lambda^{*} \notin \Lambda$. Indeed, if $\lambda^{*}=+\infty$, from the definition of $\lambda^{*}$ it is obvious that $\lambda^{*} \notin \Lambda$. Suppose that $\lambda^{*}<+\infty$ and $\lambda^{*} \in \Lambda$. Then by (14) and (21) there exists $x_{0} \leq y_{\lambda^{*}} \in P_{e}$ such that $C_{\lambda^{*}} y_{\lambda^{*}} \leq y_{\lambda^{*}}$. Similarly to the proof of (20), we have

$$
\left(A y_{\lambda^{*}}\right)(t) \leq\left(\frac{1}{2} \int_{0}^{1} \frac{s f\left(s,\left\|y_{\lambda^{*}}\right\|\right)}{x_{0}(1)} d s\right) x_{0}(t) \leq\left(\frac{1}{2} \int_{0}^{1} \frac{s f\left(s,\left\|y_{\lambda^{*}}\right\|\right)}{x_{0}(1)} d s\right)\left(B y_{\lambda^{*}}\right)(t) .
$$

Denote $\rho_{1}=\frac{1}{2} \int_{0}^{1} \frac{s f\left(s,\left\|y_{\lambda^{*}}\right\|\right)}{x_{0}(1)} d s$, then

$$
0<\rho_{1}<+\infty \text { and } A y_{\lambda^{*}} \leq \rho_{1} B y_{\lambda^{*}}
$$

Set $v=s_{1} y_{\lambda^{*}}$ for given $s_{1}>1$, then $v \in P_{e}$. Since $s_{1}^{\alpha\left(\frac{1}{s_{1}}\right)-1}<1$, we can choose $\delta>0$ such that $s_{1}^{\alpha\left(\frac{1}{s_{1}}\right)-1}<1-\delta \rho_{1}$. Therefore, from (22) we have

$$
\begin{aligned}
C_{\lambda^{*}+\delta}(v) & \leq\left(\lambda^{*}+\delta\right) s_{1} A y_{\lambda^{*}+s_{1}^{\alpha\left(\frac{1}{s_{1}}\right)} B y_{\lambda^{*}}} \\
& \leq s_{1}\left(\lambda^{*} A y_{\lambda^{*}}+\delta \rho_{1} B y_{\lambda^{*}}+B y_{\lambda^{*}}-\delta \rho_{1} B y_{\lambda^{*}}\right) \leq \nu .
\end{aligned}
$$

This means that $\lambda^{*}+\delta \in \Delta$, which is a contradiction to the definition of $\lambda^{*}$. So, $\Delta=\left[0, \lambda^{*}\right)$. Consequently, an application of Lemma 4.3 completes the proof.

In what follows, we assume that $x_{0}$ is the unique fixed point of $B$ in $P_{e}, x_{\lambda}$ is the unique fixed point of $C_{\lambda}$ in $P_{e}$ and $\lambda^{*}=\sup \Delta$. 
Theorem 4.5 Assume that (H1), (H2), (H7), and (H8) hold. Then $x_{\lambda}$ depends upon the parameter $\lambda$ as follows:

(i) $x_{\lambda}$ is nondecreasing with respect to $\lambda$ for $\lambda \in\left[0, \lambda^{*}\right)$;

(ii) $x_{\lambda}$ is continuous with respect to $\lambda$ for $\lambda \in\left[0, \lambda^{*}\right)$;

(iii) $\lim _{\lambda \rightarrow 0+}\left\|x_{\lambda}-x_{0}\right\|=0$ and $\lim _{\lambda \rightarrow \lambda^{*}-0}\left\|x_{\lambda}\right\|=+\infty$.

Proof (i) Let $\lambda_{1}, \lambda_{2} \in\left[0, \lambda^{*}\right)$ with $\lambda_{1} \leq \lambda_{2}$. Since $C_{\lambda_{1}} x_{\lambda_{2}} \leq C_{\lambda_{2}} x_{\lambda_{2}}=x_{\lambda_{2}}$, from the proof of Lemma 4.3, we find that the unique fixed $x_{\lambda_{1}}$ of $C_{\lambda_{1}}$ belongs to $\left[x_{0}, x_{\lambda_{2}}\right]$, which means that $x_{\lambda_{1}} \leq x_{\lambda_{2}}$.

(ii) Let $\lambda_{0} \in\left(0, \lambda^{*}\right)$. In order to prove $\lim _{\lambda \rightarrow \lambda_{0}^{-}}\left\|x_{\lambda_{0}}-x_{\lambda}\right\|=0$, let sequence $\left\{\lambda_{n}\right\}$ satisfy

$$
0<\lambda_{1} \leq \lambda_{2} \leq \cdots \leq \lambda_{n} \leq \cdots \leq \lambda_{0} \text { and } \lim _{n \rightarrow+\infty} \lambda_{n}=\lambda_{0}
$$

By virtue of the above conclusion (i) we have

$$
x_{\lambda_{1}} \leq x_{\lambda_{2}} \leq \cdots \leq x_{\lambda_{n}} \leq \cdots \leq x_{\lambda_{0}}
$$

which implies that $\left\{x_{\lambda_{n}}\right\}$ is a bounded subset in $P$. Further, similarly to the proof of the conclusion (ii) in Lemma 3.3 we see that $\left\{x_{\lambda_{n}}\right\}$ converges to $x^{*} \in P$. From (23) we have $x^{*} \in\left[x_{\lambda_{1}}, x_{\lambda_{0}}\right]$, which leads to $x^{*} \in P_{e}$. Note that

$$
x_{\lambda_{n}}=\lambda_{n} A x_{\lambda_{n}}+B x_{\lambda_{n}} .
$$

By taking the limit we have $x^{*}=\lambda_{0} A x^{*}+B x^{*}=C_{\lambda_{0}} x^{*}$. Since $C_{\lambda_{0}}$ has only one fixed point in $P_{e}$, then $x^{*}=x_{\lambda_{0}}$. This means that $\left\|x_{\lambda_{0}}-x_{\lambda}\right\| \rightarrow 0$ as $\lambda \rightarrow \lambda_{0}^{-}$.

A similar argument can show that for any $\lambda_{0} \in\left[0, \lambda^{*}\right),\left\|x_{\lambda}-x_{\lambda_{0}}\right\| \rightarrow 0$ as $\lambda \rightarrow \lambda_{0}^{+}$. Thus, the proof of (ii) is complete.

(iii) It is obvious from the above conclusion (ii) that $\lim _{\lambda \rightarrow 0+}\left\|x_{\lambda}-x_{0}\right\|=0$.

In order to finish the proof of $\lim _{\lambda \rightarrow \lambda^{*}-0}\left\|x_{\lambda}\right\|=+\infty$, we consider two cases.

Case 1. $\lambda^{*}=+\infty$.

Since $x_{\lambda}=\lambda A x_{\lambda}+B x_{\lambda} \geq \lambda A x_{0}$, then $\left\|x_{\lambda}\right\| \geq \lambda\left\|A x_{0}\right\|$, which means $\lim _{\lambda \rightarrow \lambda^{*}-0}\left\|x_{\lambda}\right\|=+\infty$.

Case 2. $\lambda^{*}<+\infty$

By the above conclusion (i) we have $\lim _{\lambda \rightarrow \lambda^{*}-0}\left\|x_{\lambda}\right\| \leq+\infty$. Suppose to the contrary that $\lim _{\lambda \rightarrow \lambda^{*}-0}\left\|x_{\lambda}\right\|<+\infty$. Similarly to the case 2 in the proof of Lemma 3.3, we conclude that $C_{\lambda^{*}}$ has a fixed point $x^{*} \in P \backslash\{\theta\}$. From Remark 4.3 we have $x^{*} \in P_{e}$. So $\lambda^{*} \in\left[0, \lambda^{*}\right)$, which is a contradiction. This ends the proof.

Now, we give an estimate for critical value $\lambda^{*}$ in Theorem 4.4. If (H1) and (H8) hold, then

$$
\frac{f(t, x)}{x} \leq f(t, 1) \leq \max _{t \in[0,1]} f(t, 1), \quad x>1, t \in[0,1] .
$$

Moreover, $F_{\infty}=\lim \sup _{x \rightarrow+\infty} \max _{t \in[0,1]} \frac{f(t, x)}{x} \in[0,+\infty)$.

Theorem 4.6 Assume that (H1), (H2), (H7), and (H8) hold. Then

$$
\lambda^{*} \begin{cases}\geq \frac{2}{F_{\infty}}, & 0<F_{\infty}<+\infty \\ =+\infty, & F_{\infty}=0\end{cases}
$$


Proof For any $\epsilon>0$, there exists $r_{0} \in(0,1)$ such that

$$
q(1) \leq \frac{1}{r_{0}} \quad \text { and } \quad f\left(t, \frac{1}{r}\right) \leq \frac{1}{r}\left(F_{\infty}+\epsilon\right), \quad r \leq r_{0}, t \in[0,1]
$$

Note that $\frac{r_{0}^{\alpha\left(r_{0}\right)}}{r_{0}}>1$, we can choose a sufficiently large positive integer number $k$ such that $\left(\frac{r_{0}^{\alpha\left(r_{0}\right)}}{r_{0}}\right)^{k} \geq \frac{1}{r_{0}}$, that is,

$$
\left(\frac{1}{r_{0}^{\alpha\left(r_{0}\right)}}\right)^{k} \leq \frac{1}{r_{0}^{k-1}}
$$

Let $w(t)=\left(\frac{1}{r_{0}}\right)^{k} e(t)$, then, from (4), (25), and (26) we have

$$
\begin{aligned}
& (A w)(t) \leq \frac{t^{2}}{2} \int_{0}^{1} s f\left(s, \frac{1}{r_{0}^{k}}\right) d s \leq \frac{t^{2}}{2 r_{0}^{k-1}} \int_{0}^{1} s f\left(s, \frac{1}{r_{0}}\right) d s \leq \frac{1}{4}\left(F_{\infty}+\epsilon\right) w(t), \\
& (B w)(t) \leq \frac{t^{2}}{2} q\left(\frac{1}{r_{0}^{k}}\right) \leq \frac{t^{2}}{2}\left(\frac{1}{r_{0}^{\alpha\left(r_{0}\right)}}\right) q\left(\frac{1}{r_{0}^{k-1}}\right) \leq \frac{t^{2}}{2}\left(\frac{1}{r_{0}^{\alpha\left(r_{0}\right)}}\right)^{k} q(1) \leq \frac{t^{2}}{2}\left(\frac{1}{r_{0}}\right)^{k}=\frac{1}{2} w(t) .
\end{aligned}
$$

Moreover, taking $\lambda_{\epsilon}=\frac{2}{F_{\infty}+\epsilon}$, we have

$$
\left(C_{\lambda_{\epsilon}} w\right)(t)=\lambda_{\epsilon}(A w)(t)+(B w)(t) \leq \frac{1}{4} \lambda_{\epsilon}\left(F_{\infty}+\epsilon\right) w(t)+\frac{1}{2} w(t) \leq w(t)
$$

Consequently, from (21) we obtain $\lambda_{\epsilon}=\frac{2}{F_{\infty}+\epsilon} \in\left[0, \lambda^{*}\right)$, that is, $\lambda^{*}>\frac{2}{F_{\infty}+\epsilon}$, which implies that (24) holds. This completes the proof.

Remark 4.4 Different from Theorems 3.2 and 3.4, the estimate of $\lambda^{*}$ in Theorem 4.6 does not take into account effect of $q(x)$. This is valuable, because the conditions (H2) and (H7) cannot ensure $Q_{\infty}<2$ as $\lim \sup _{r \rightarrow 0} \alpha(r)=1$. Certainly, if $Q_{\infty}<2$, then $\lambda^{*} \geq \frac{2\left(2-Q_{\infty}\right)}{F_{\infty}}$. In particular, if $Q_{\infty}=0$, then $\lambda^{*} \geq \frac{4}{F_{\infty}}$.

Corollary 4.7 Assume that (H1), (H2), (H7), and (H9) hold. Then

(i) BVP (1) has a unique positive solution $x_{\lambda}$ in $P_{e}$ for $\lambda \in[0,+\infty)$. Moreover, for any $w_{0} \in P_{e}$, set $w_{n}=\lambda A w_{n-1}+B w_{n-1}(n=1,2, \ldots)$, then $\lim _{n \rightarrow+\infty}\left\|w_{n}-x_{\lambda}\right\|=0$;

(ii) $x_{\lambda}$ is nondecreasing with respect to $\lambda$ for $\lambda \in[0,+\infty)$;

(iii) $x_{\lambda}$ is continuous with respect to $\lambda$ for $\lambda \in[0,+\infty)$;

(iv) $\lim _{\lambda \rightarrow 0+}\left\|x_{\lambda}-x_{0}\right\|=0$ and $\lim _{\lambda \rightarrow+\infty}\left\|x_{\lambda}\right\|=+\infty$.

Proof From (H1), (H2), (H7), and (4), we see that $C_{\lambda}: P_{e} \rightarrow P_{e}$ is increasing for any given $\lambda \geq 0$. Further, for any given $\lambda \geq 0$ we have

$$
C_{\lambda}(r x)=\lambda A(r x)+B(r x) \geq r^{\delta(r)} C_{\lambda} x, \quad x \in P_{e}, r \in(0,1),
$$

where $\delta(r)=\max \{\alpha(r), \beta(r)\}$. Thus, the conclusion (i) follows from Lemma 1.2.

From (H9), we have $f(t, r x) \geq r f(t, x)$ for $r \in(0,1), t \in[0,1]$ and $x \in[0,+\infty)$. Therefore, in the same way as in the proof of Theorem 4.5, we can complete the rest of the proof. 
When $q(x) \equiv c>0$ is a constant function, $Q_{\infty}=0$ and $B x(t)=c\left(\frac{1}{2} t^{2}-\frac{1}{6} t^{3}\right):=x_{0}(t)$. It is evident that $B$ satisfies (H2) and (H7). So we can obtain the following two results.

Corollary 4.8 Assume that (H1) and (H8) hold. If $F_{\infty}>0$, then

(i) there exists $\lambda^{*} \geq \frac{4}{F_{\infty}}>0$ such that $B V P$ (1) with $q(x) \equiv c$ has a unique positive solution $x_{\lambda}$ in $P_{e}$ for $\lambda \in\left[0, \lambda^{*}\right)$ and does not have any solution in $P_{e}$ for $\lambda \geq \lambda^{*}$. Moreover, for any $w_{0} \in P_{e}$, set $w_{n}=x_{0}+\lambda A w_{n-1}(n=1,2, \ldots)$, then $\lim _{n \rightarrow+\infty}\left\|w_{n}-x_{\lambda}\right\|=0$;

(ii) $x_{\lambda}$ is nondecreasing with respect to $\lambda$ for $\lambda \in\left[0, \lambda^{*}\right)$;

(iii) $x_{\lambda}$ is continuous with respect to $\lambda$ for $\lambda \in\left[0, \lambda^{*}\right)$;

(iv) $\lim _{\lambda \rightarrow 0+}\left\|x_{\lambda}-x_{0}\right\|=0$ and $\lim _{\lambda \rightarrow \lambda^{*}-}\left\|x_{\lambda}\right\|=+\infty$.

Corollary 4.9 Assume that $(\mathrm{H} 1)$ and $(\mathrm{H} 8)$ hold. If $F_{\infty}=0$, then

(i) for any $\lambda \in[0,+\infty), B V P(1)$ with $q(x) \equiv c$ has a unique positive solution $x_{\lambda}$ in $P_{e}$, moreover, for any $w_{0} \in P_{e}$, set $w_{n}=x_{0}+\lambda A w_{n-1}(n=1,2, \ldots)$, then $\lim _{n \rightarrow+\infty}\left\|w_{n}-x_{\lambda}\right\|=0$;

(ii) $x_{\lambda}$ is nondecreasing in $\lambda$ for $\lambda \in[0,+\infty)$;

(iii) $x_{\lambda}$ is continuous with respect to $\lambda$ for $\lambda \in[0,+\infty)$;

(iv) $\lim _{\lambda \rightarrow 0+}\left\|x_{\lambda}-x_{0}\right\|=0$ and $\lim _{\lambda \rightarrow+\infty}\left\|x_{\lambda}\right\|=+\infty$.

Corollary 4.10 Assume that (H1) and (H9) hold. Then the conclusions (i), (ii), (iii), and (iv) in Corollary 4.9 hold.

Finally, we give two concrete examples to illustrate those results in the section.

Example 1 In BVP (1), let

$$
f(t, x)=\left\{\begin{array}{ll}
\frac{t}{3} x, & 0 \leq x \leq 1, \\
\frac{t}{6}(x+\sqrt{x}), & x>1
\end{array} \quad \text { and } \quad q(x)= \begin{cases}x^{\frac{3}{5}}, & 0 \leq x \leq 32 \\
8, & x>32\end{cases}\right.
$$

it is obvious that the conditions (H1) and (H2) are satisfied. For any $r \in(0,1)$,

as $0 \leq x \leq 1$, we have $f(t, r x)=\frac{t r}{3} x=r f(t, x)$;

as $x>1$ and $0<r x \leq 1$, we have $f(t, r x)=\frac{t r}{3} x \geq \frac{r t}{3}\left(\frac{1}{2} x+\frac{1}{2} \sqrt{x}\right) \geq \frac{t r}{6}(x+\sqrt{x})=r f(t, x)$;

as $x>1$ and $r x>1$, we have $f(t, r x)=\frac{t}{6}(r x+\sqrt{r x}) \geq \frac{t r}{6}\left(x+\frac{1}{\sqrt{r}} \sqrt{x}\right) \geq r f(t, x)$, that is, $f(t, r x) \geq r f(t, x)$ for $x \in[0,+\infty)$ and $t \in[0,1]$. Similarly, we can obtain $q(r x) \geq$ $r^{\frac{3}{5}} q(x)$ for $x \in[0,+\infty)$. Therefore, the conditions (H7) and (H8) are satisfied. Note that

$$
F_{\infty}=\limsup _{x \rightarrow+\infty} \max _{t \in[0,1]} \frac{f(t, x)}{x}=\frac{1}{6}, \quad Q_{\infty}=\limsup _{x \rightarrow+\infty} \frac{q(x)}{x}=0 .
$$

By Theorems 4.4, 4.5 and Remarks 4.3, 4.4 we see that there exists $\lambda^{*} \geq 24$ such that BVP (1) has a unique positive solution $x_{\lambda}$ for $\lambda \in\left[0, \lambda^{*}\right)$ and does not have any positive solution for $\lambda \geq \lambda^{*}$. Moreover, for any $w_{0} \in P_{e}$, set $w_{n}(t)=\lambda A w_{n-1}(t)+B w_{n-1}(t)(n=1,2, \ldots)$, then $\lim _{n \rightarrow \infty}\left\|w_{n}-x_{\lambda}\right\|=0$, and such solution $x_{\lambda}(t)$ satisfies the properties (i), (ii), and (iii) in Theorem 4.5 . 


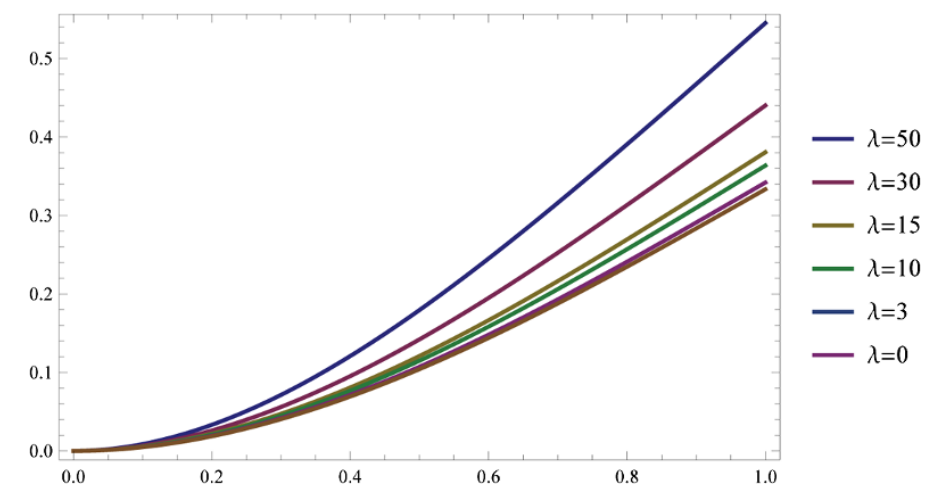

Figure 1 Solutions for BVP (1) with $f(t, x)=\frac{t(1-t) x}{1+x}$ and $q(x)=1$

Example 2 In BVP (1), let $f(t, x)=\frac{t(1-t) x}{1+x}, q(x)=1, t \in[0,1], x \in[0,+\infty)$, it is easy to see that $(\mathrm{H} 1)$ holds. For any $r \in(0,1)$, we have

$$
f(r x)=\frac{t(1-t) r x}{1+r x} \geq r \cdot \frac{t(1-t) x}{1+x} \geq r f(t, x), \quad x \in[0,+\infty) .
$$

So, (H8) holds. Note that

$$
F_{\infty}=\limsup _{x \rightarrow+\infty} \max _{t \in[0,1]} \frac{f(t, x)}{x}=0,
$$

by Corollary 4.9 we find that BVP (1) has a unique positive solution $x_{\lambda}$ for $\lambda \geq 0$. Moreover, for any $w_{0} \in P_{e}$, set $w_{n}(t)=\frac{1}{2} t^{2}-\frac{1}{6} t^{3}+\lambda A w_{n-1}(t)(n=1,2, \ldots)$, then $\lim _{n \rightarrow \infty}\left\|w_{n}-x_{\lambda}\right\|=0$, and such a solution $x_{\lambda}(t)$ satisfies the properties (ii), (iii), and (iv) in Corollary 4.9 with $x_{0}(t)=\frac{1}{2} t^{2}-\frac{1}{6} t^{3}$.

In this example, by using Wolfram Mathematica 9.0, we can plot the graphs of solutions $x_{\lambda}(t)$ for BVP (1) with $\lambda=0,3,10,15,30,50$, as the Figure 1 shows.

\section{Competing interests}

The authors declare that they have no competing interests.

\section{Authors' contributions}

All authors participated in drafting, revising and commenting on the manuscript. All authors read and approved the final manuscript.

\section{Acknowledgements}

The authors sincerely thank the reviewers for their valuable suggestions and useful comments. This research was supported by the NNSF of China (11361047), the University Natural Science Research Develop Foundation of Shanxi Province of China (20111021, 2013156), Research Project Supported by Shanxi Scholarship Council of China (2013-102) and the Science Foundation of Qinghai Province of China (2012-Z-910).

Received: 7 October 2013 Accepted: 26 March 2014 Published: 09 Apr 2014

\section{References}

1. Aftabizadeh, AR: Existence and uniqueness theorems for fourth-order boundary value problems. J. Math. Anal. Appl. $116,415-426(1986)$

2. Agarwal, RP, Chow, YM: Iterative methods for a fourth order boundary value problem. J. Comput. Appl. Math. 10, 203-217 (1984)

3. Gupta, CP: Existence and uniqueness results for a bending of an elastic beam equation at resonance. J. Math. Anal. Appl. 135, 208-225 (1988)

4. Bai, Z, Wang, H: On positive solutions of some nonlinear fourth-order beam equations. J. Math. Anal. Appl. 270, 357-368 (2002) 
5. Bai, Z: The upper and lower solution method for some fourth-order boundary value problems. Nonlinear Anal. 67(6), 1704-1709 (2007)

6. Bonanno, G, Bellaa, BD: A boundary value problem for fourth-order elastic beam equations. J. Math. Anal. Appl. 343, 1166-1176 (2008)

7. Li, Y: Positive solutions of fourth-order boundary value problems with two parameters. J. Math. Anal. Appl. 281, 477-484 (2003)

8. Liu, XL, Li, WT: Positive solutions of the nonlinear fourth-order beam equation with three parameters. J. Math. Anal. Appl. 303, 150-163 (2005)

9. Liu, B: Positive solutions of fourth-order two point boundary value problems. Appl. Math. Comput. 148, 407-420 (2004)

10. Ma, RY, Xu, L: Existence of positive solutions of a nonlinear fourth-order boundary value problem. Appl. Math. Lett. 23, 537-543 (2010)

11. Ma, RY, Thompson, B: Nodal solutions for a nonlinear fourth-order eigenvalue problem. Acta Math. Sin. Engl. Ser. 24(1), 27-34 (2008)

12. Yang, B: Positive solutions for a fourth order boundary value problem. Electron. J. Qual. Theory Differ. Equ. 2005(3), 1-17 (2005)

13. Zhang, X: Existence and iteration of monotone positive solutions for an elastic beam with a corner. Nonlinear Anal., Real World Appl. 10, 2097-2103 (2009)

14. Graef, JR, Qian, C, Yang, B: A three point boundary value problem for nonlinear fourth order differential equations. J. Math. Anal. Appl. 287, 217-233 (2003)

15. Yao, QL: Existence and multiplicity of positive solutions to a singular elastic beam equation rigidly fixed at both ends. Nonlinear Anal. 69, 2683-2694 (2008)

16. Yao, QL: Local existence of multiple positive solutions to a singular cantilever beam equation. J. Math. Anal. Appl. 363 138-154 (2010)

17. Ma, TF: Positive solutions for a beam equation on a nonlinear elastic foundation. Math. Comput. Model. 39, 1195-1201 (2004)

18. Li, SY, Zhang, XQ: Existence and uniqueness of monotone positive solutions for an elastic beam equation with nonlinear boundary conditions. Comput. Math. Appl. 63, 1355-1360 (2012)

19. Alves, E, Ma, TF, Pelicer, ML: Monotone positive solutions for a fourth order equation with nonlinear boundary conditions. Nonlinear Anal. 71, 3834-3841 (2009)

20. Amann, H: Fixed point equations and nonlinear eigenvalue problems in ordered Banach spaces. SIAM Rev. 18, 602-709 (1976)

21. Guo, DJ, Lakshmikantham, V: Nonlinear Problems in Abstracts Cone. Academic Press, New York (1988)

22. Deimling, K: Nonlinear Functional Analysis. Springer, Berlin (1985)

23. Zhai, CB, Yang, C, Guo, CM: Positive solutions of operator equations on ordered Banach spaces and applications. Comput. Math. Appl. 56, 3150-3156 (2008)

10.1186/1687-2770-2014-80

Cite this article as: Wang et al.: Positive solutions for elastic beam equations with nonlinear boundary conditions and a parameter. Boundary Value Problems 2014, 2014:80

\section{Submit your manuscript to a SpringerOpen ${ }^{\circ}$ journal and benefit from:}

- Convenient online submission

Rigorous peer review

- Immediate publication on acceptance

- Open access: articles freely available online

- High visibility within the field

- Retaining the copyright to your article 